\title{
CONSUMO DE ALIMENTOS CONFORME 0 GRAU DE PROCESSAMENTO E A AVALIAÇÃO NUTRICIONAL DE MULHERES GAÚCHAS
}

Carolina Pagnoncelli Gabrielli; Universidade Federal do Paraná; carolinapgabrielli@gmail.com Simone Bonatto; Universidade de Caxias do Sul; sbonatto1@ucs.br Josiane Siviero; Universidade de Caxias do Sul; jsiviero@ucs.br

\section{RESUMO}

Introdução: Ao longo da vida são estabelecidas preferências alimentares, predominando escolhas alimentares mais palatáveis a cada indivíduo sendo na maioria das vezes, alimentos industrializados. Essa composição alimentar ingerida diariamente e em grande quantidade, reflete no aumento do excesso de peso e da prevalência de outras doenças crônicas. Objetivo: avaliar as preferências alimentares, conforme o grau de processamento de alimentos e relacionar com a avaliação nutricional de mulheres moradoras da Serra Gaúcha. Método: Trata-se de um estudo transversal retrospectivo. A amostra foi composta por 204 mulheres com idade $\geq 50$ anos. As variáveis demográficas analisadas foram: idade, estado civil, renda e escolaridade. Quanto a avaliação nutricional, foram consideradas circunferência da cintura (CC) e índice de massa corporal (IMC), e o consumo alimentar foi avaliado mediante a questionário de frequência alimentar (QFA). A análise estatística foi através do software Statistical Package for the Social Sciences (SPSS). Resultados: Houve prevalência de excesso de peso e risco para doenças cardiovasculares (DCV). Quanto ao consumo alimentar, verificou-se que as entrevistadas que apresentavam excesso de peso e risco para DCV, ingeriam alimentos in natura e/ou minimamente processados em menor quantidade em comparação às eutróficas. Conclusão: $\mathrm{O}$ consumo dessa categoria de processamento de alimentos é um fator de proteção à obesidade e outras doenças crônicas, porém mais estudos são essenciais para evidenciar ainda mais este benefício.

Palavras-chave: Estado nutricional; Alimentos processados; Envelhecimento 